\title{
Malignant inflammatory fibrous histiocytoma of bone and acute lymphoblastic leukaemia
}

\author{
B.E. Woodcock ${ }^{1}$, M.A. Parsons ${ }^{2}$ and D.A. Winfield ${ }^{1}$ \\ Departments of ${ }^{1}$ Haematology and ${ }^{2}$ Pathology, The Royal Hallamshire Hospital and the University of Sheffield, \\ Sheffield, UK.
}

\begin{abstract}
Summary: A patient is described who developed a malignant inflammatory fibrous histiocytoma of vertebral bone (MFH) 4 months after the onset of acute lymphoblastic leukaemia. This is the first report of the simultaneous occurrence of these two conditions. Similarities to the more frequent occurrence of another histiocytic malignancy, histiocytic medullary reticulosis, following acute lymphoblastic leukaemia, are noted. The usefulness of gallium scanning to monitor the response of this tumour to treatment is demonstrated.
\end{abstract}

\section{Case report}

A 29 year old male Caucasian instrument mechanic presented with a history of a headache, malaise and indigestion of 1 week's duration. Ten days previously he noticed swellings in the right side of his neck and he began to sweat at night. On examination there was moderate enlargement of the cervical and axillary lymph nodes. There were no other physical abnormalities. A peripheral blood count showed a haemoglobin of $15.2 \mathrm{~g} / \mathrm{dl}$, a leucocyte count of $28.4 \times 10^{9} / 1$ (lymphoblasts $\left.19.3 \times 10^{9} / 1\right)$ and a platelet count of $38 \times 10^{9} / 1$. A bone marrow aspirate showed a hypercellular marrow replaced by typical lymphoblasts with negative stains for PAS, peroxidase and combined esterase. Thirty per cent of the lymphoblasts contained acid phosphatase positive granules, which appeared randomly distributed. Eighty seven per cent of the cells carried the common-ALL antigen and $50 \%$ were positive for terminal deoxynucleotidyl transferase. A diagnosis of acute lymphoblastic leukaemia (ALL) was made.

The patient was entered into the current Medical Research Council Trial (UKALL IX) and drew schedule ' $D$ '. Remission was achieved at 4 weeks using daunorubicin, vincristine, prednisolone and erwinia asparaginase. Prophylactic central nervous system treatment comprised cranial irradiation $(1800 \mathrm{cGy}$ in divided doses) and intrathecal methotrexate, during which oral mercaptopurine was given. A bone marrow

Correspondence: B.E. Woodcock, M.R.C.P. Department of Haematology, Royal Hallamshire Hospital, Sheffield S10 2JF

Accepted: 28 November 1984 aspirate at week 12 confirmed remission, and maintenance treatment continued with vincristine and prednisolone followed by $30 \mathrm{mg}$ methotrexate for $3 \mathrm{~d}$ and $150 \mathrm{mg} / \mathrm{d}$ mercaptopurine for three weeks.

Nineteen weeks after anti-leukaemia therapy had begun the patient was re-admitted to hospital complaining of severe back pain of 2 weeks duration. He was nauseated, vomiting and restless and had a temperature of $38.5^{\circ} \mathrm{C}$. There were no other physical examination findings. X-ray of his lumbar spine and computed tomographic brain scan were normal; an attempted lumbar puncture was unsuccessful. A gallium isotope scan showed an area of increased uptake at the $L_{1} / L_{2}$ region of the lumbar spine. A provisional diagnosis of paravertebral abscess was made, and he was treated with broad spectrum antibiotic therapy with return to normal of his temperature. Four weeks later, despite continuing antibiotic therapy, he was re-admitted with a recurrent fever and increasing back pain. Plain X-ray of the lumbar spine showed destruction of the right lateral part of the vertebral body, the pedicle and the transverse process of $\mathrm{L}_{1}$ (Figure 1). A repeat gallium scan showed that the area of increased uptake had increased in size. Surgical exploration revealed a tumour which was only partially resectable.

Histologically the tumour consisted of mononuclear and multinucleated tumour cells admixed with neutrophils and lymphocytes (Figure 2). The findings are those of malignant inflammatory fibrous histiocytoma as described by Kyriakos \& Kempson (1976), and are supported by electron microscopic and immunocytochemical findings.

Treatment consisted of radiotherapy to the tumour 


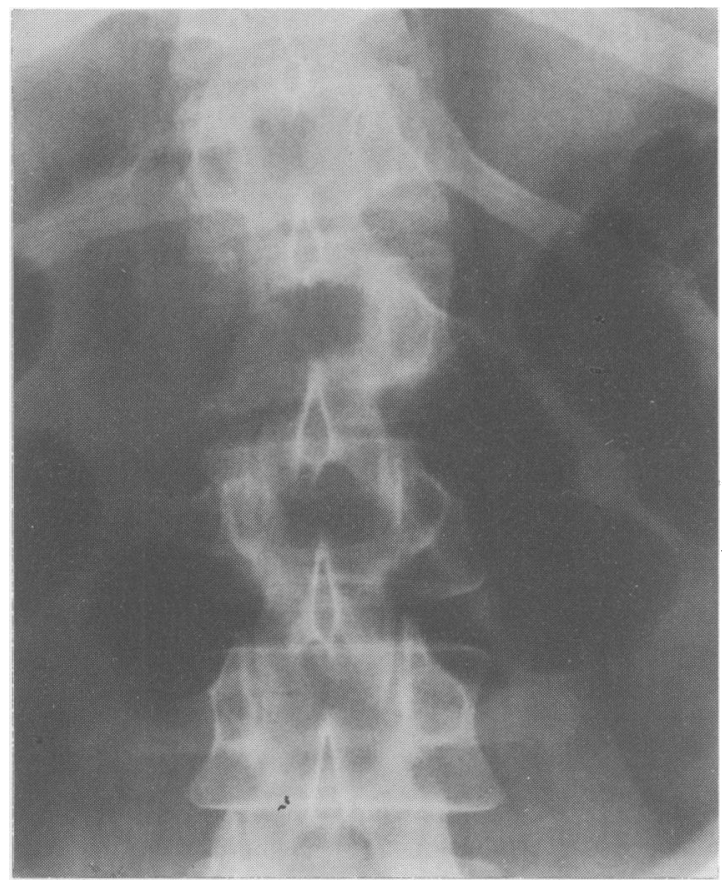

Figure 1 Destruction of the right lateral part of the vertebral body, pedicle and transverse process of $L_{1}$.

site and chemotherapy, with methotrexate, cyclophosphamide, adriamycin and actinomycin.

Eight months after the diagnosis of malignant fibrous histiocytoma he developed severe pain in the lower right leg which became swollen, hot and tender below the right knee. Plain X-ray demonstrated a lytic lesion in the lower end of the right fibula and a gallium scan showed a diffuse increase in uptake over both knees and ankles. A biopsy of both upper and lower lesions of the right leg confirmed the presence of malignant fibrous histiocytoma histologically identical to the primary tumour. Local radiotherapy was symptomatically effective and the patient continues in haematological remission 23 months from diagnosis. There is at present no increase in uptake of gallium in his lumbar spine and X-rays of the lumbar spine show healing along the margin of the resected tumour.

\section{Discussion}

We believe this to be the first reported case of malignant fibrous histiocytoma (MFH) occurring in a patient with acute lymphoblastic leukaemia. Three factors suggest that this was not due to chance. Firstly, the close temporal association of the two conditions, with MFH presenting only 4 months after a diagnosis

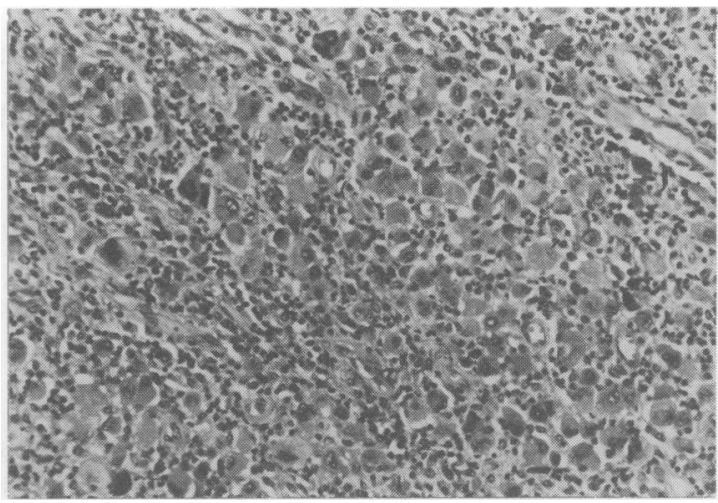

Figure 2 Photomicrograph of the vertebral tumour. The malignant fibrous histiocytoma is composed of bizarre multinucleated giant cells and large mononuclear tumour cells, admixed with lymphocytes and neutrophil polymorphs (H \& E × 257).

of ALL was made. Secondly, although MFH is a comparatively common sarcoma in the elderly, less than $10 \%$ of cases occur under the age of 30 years. Finally, MFH has been reported to occur in association with both malignant haematological disorders, such as Hodgkin's disease, non-Hodgkin's lymphoma, multiple myeloma, myeloblastic leukaemia, and malignant histiocytosis (Weiss \& Enzinger, 1978).

Mosijczuk \& Ruymann (1981) have suggested that the frequency of second malignancy in patients with ALL is increased, although the evidence that this is due to the disease itself or the effects of its treatment is inconclusive. Zarrabi et al. (1983) identified reports of 17 patients with ALL developing acute myeloblastic leukaemia, 12 chronic myelocytic leukaemia, 19 lymphoma and 13 solid tumour. Of the 19 patients developing lymphoma, 9 developed histiocytic medullary reticulosis (HMR) and the literature now contains 13 such cases, all of which have been fatal (Liu Yin et al., 1983). The HMR has occurred within 8 months of the diagnosis of ALL whereas other nonleukaemic malignancies have not occurred earlier than 10 months from diagnosis. It is therefore striking that the malignant fibrous histiocytoma seen in our case occurred at the time of increased frequency of another histiocytic malignancy. The short period between treatment and the onset of the second malignancy in these cases suggests that chemotherapy and radiotherapy are unlikely to be the direct cause of these malignancies. It should be noted that no condition previously reported as pre-disposing to MFH, such as bone infarcts, radiotherapy or Paget's disease was present.

The MFH in this case was evidently radiosensitive as clearance of the primary tumour was demonstrated 
by gallium scanning. The emergence of distant metastases during chemotherapy indicates that the tumour was resistant to the drugs used. We emphasize the usefulness of gallium scanning in monitoring the response of the tumour to therapy and in localizing distant metastases (Zazzaro et al., 1981).

\section{References}

KYRIAKOS, M. \& KEMPSON, R.L. (1976). Inflammatory fibrous histiocytoma. An aggressive and lethal lesion. Cancer, 37, 1584.

LIU YIN, J.A., KUMARAN, T.O., MARSH, G.W., ROSSITER, M. \& CATOVSKY, D. (1983). Complete recovery of histiocytic medullary reticulosis like syndrome in a child with acute lymphoblastic leukaemia. Cancer, 51, 200.

MOSIJCZUK, A.D. \& RUYMANN, F.B. (1981). Second malignancy in acute lymphocytic leukaemia. American Journal of Diseases of Children, 135, 313.

\section{Acknowledgements}

The authors thank Dr B. W. Hancock and Dr D. Bradshaw for their advice and help in treating this patient.

WEISS, S.W. \& ENZINGER, F.M. (1978). Malignant fibrous histiocytoma. Cancer, 41, 2250.

ZARRABI, M.H., ROSNER, F. \& GRÜNWALD, H.W. (1983). Second neoplasms in acute lymphoblastic leukaemia. Cancer, 52, 1712.

ZAZZARO, P.F., BOSWORTH, J.E., SCHNEIDER, V. \& ZELENAK, J.J. (1981). Gallium scanning in malignant fibrous histiocytoma. American Journal of Roentgenology, 135, 775. 